\title{
Meta-Analysis of Human Molecular Responses to Staphylococcus aureus
}

Sidra Younis ${ }^{1}$, Farha Deeba ${ }^{2}$, Qamar Javed $^{3}$, Miroslav Blumenberg ${ }^{4}$

\section{ABSTRACT}

Objective: To compare the local and systematic transcriptional responses of human body to $S$. aureus or its components.

Study Design: Meta-analysis of microarray data.

Place and Duration of Study: The study was conducted at R.O. Perelman Department of Dermatology, The NYU Cancer Institute, NYU Langone Medical Center, New York, USA, from March 2015 to May 2015.

Materials and Methods: Public repository "GEO Datasets" was searched using key term "Staphylococcus aureus" for data sets covering effects of $S$. aureus infection in Homo sapiens cells. The microarray data for immune cell responses to $S$. aureus was analyzed using Rank Prod, RMA Express and DAVID software.

Results: The analysis has shown that $S$. aureus infection was responsible for inducing immunity, platelet activation, vasodilation, MyD88 dependent gene expression and cell cycle. It suppressed gene expression of normal cell processes, protein catabolism and apoptosis. Heat-inactivated $S$. aureus challenged the cell induced immunity, cell cycle, growth regulators, anti-apoptosis and anticoagulant genes, while suppressed the genes for adaptive immunity, carbohydrate synthesis and Myd88 dependent pathway. Furthermore, in the $S$. aureus-infected patients the genes for defense, innate immunity, solute receptors and anti-apoptotic processes were upregulated, whereas adaptive immunity and positive regulators of apoptosis were downregulated.

Conclusion: MyD88 signaling pathway, ubiquitin mediated protein catabolism and IFNY mediated cell death processes can be targeted for treatment against virulent S. aureus infections.

\section{Key Words: Heat-killed S. aureus, Immunity, Live S. aureus, Meta-Analysis, Staphylococcus aureus.}

How to cite this: Younis S, Deeba F, Javed Q, Blumenberg M. Meta-Analysis of Human Molecular Responses to Staphylococcus aureus. Life and Science. 2020; 1(4): 128-138. doi: http://doi.org/10.37185/LnS.1.1.104

This is an Open Access article distributed under the terms of the Creative Commons Attribution License (http://creativecommons.org/licenses/by/4.0), which permits unrestricted use, distribution, and reproduction in any medium, provided the original work is properly cited.

\section{Introduction}

Staphylococcus aureus (S. aureus) is a gram-positive bacterium found as part of normal flora in $50 \%$ of population. It is a common cause of skin infections

\footnotetext{
${ }^{1}$ Department of Biological Sciences

National University of Medical Sciences, Rawalpindi

${ }^{2}$ Department of Biochemistry and Biotechnology

The Women University, Multan

${ }^{3}$ Department of Biochemistry

Quaid-i-Azam University, Islamabad

${ }^{4}$ R.O. Perelman Department of Dermatology

The NYU Cancer Institute

NYU Langone Medical Center, New York, USA

Correspondence:

Dr. Sidra Younis

Assistant Professor, Biological Sciences

National University of Medical Sciences, Rawalpindi

E-mail: sidra.younis@numspak.edu.pk

Funding Source: HEC; Conflict of Interest: NIL

Received: Mar 03, 2020; Revised: May 11, 2020

Accepted: Sep 08, 2020
}

including impetigo, folliculitis, cellulitis, infected ulcers, wounds, respiratory diseases and food poisoning. The severity of $S$. aureus infections can range from mild local to severe deeper soft tissue infections requiring hospitalization. Many antibiotics have been designed to target the bacterial wall synthesis. However, antibiotic-resistant forms of pathogenic $S$. aureus, e.g. methicillin-resistant $S$. aureus (MRSA), have become a widespread clinical problem. S. aureus produce enzymes that protect it from degradation and have acquired two types of strategies to avoid killing by host immune cells. $S$. aureus infection activates cell growth and metabolic pathways, which provides a rich growth environment. It activates anti-apoptotic protein transcription to decrease apoptosis of the infected cells. ${ }^{1}$

S. aureus and associated toxins are recognized by a 
number of surface and sub-cellular receptors in host cells, such as Toll-like receptor (TLR), retinoic acidinducible gene-I-like receptors (RIG) and nucleotidebinding oligomerization domain-like receptors (NOD) like receptors. The activation of these receptors and co-stimulatory molecules results in cascades of reactions which lead to activation of nuclear factor kappa-light-chain-enhancer of activated B cells (NFkB) or interferon gamma (IFNG) through Myeloid differentiation primary response 88 (MyD88)-dependent or independent pathways. Subsequent production of cytokines, chemokines and complement molecules activate either innate or adaptive immune system, based on the triggering factors. Additionally, pathogen containing phagosomes are processed by endoplasmic reticulum and presented as peptides on the surface of antigen presenting cells as a complex with major histocompatibility molecules ( $\mathrm{MHCl}$ or $\mathrm{MHClI}$ ) or heat shock proteins. Some heat shock proteins can process pathogen specific peptides independent of endoplasmic reticulum and Golgi apparatus, thus giving extra advantages in antigen presentation and activation of innate immune specific proteins and pathways. ${ }^{2,3,4}$

Although considerable information is available about $S$. aureus virulence, key pathways or signaling molecules are still obscure. To explore this problem, we carried out a meta-analysis of freely available data in public repositories to compare the local and systematic transcriptional responses of human body to $S$. aureus or its components.

\section{Materials and Methods}

We have compared transcription differences between untreated, control cells versus those challenged with active $S$. aureus, inactive $S$. aureus and S. aureus infected patients' blood.

\section{Search Strategy}

Public repository "GEO Datasets" was searched using key term "Staphylococcus aureus" for data sets covering effects of $S$. aureus infection in Homo sapiens cells. In these studies, effects of live heat- or UV-inactivated $S$. aureus cultures were studied in epithelial or immune cells. The cells were treated for different time periods. In 3 data sets differential expression in blood drawn from infected patients and healthy controls was studied. (Fig 1)

Analysis of differential expression in cells infected

\section{with live S. aureus}

Data from 6 studies, 9 datasets, 214 microarrays and 21,632 genes were combined. In these studies, $S$. aureus infection responses were observed in macrophages, neutrophils, leukocytes, human umbilical vein endothelial cells and a hepatocellular carcinoma cell line. Affymetrix and Illumina platforms were used for differential expression analysis in infected and control cells. For this analysis, one data set was compromised; the first due to missing data and the second for fewer common genes with other data sets (Table 1 and Fig 2).

\begin{tabular}{|c|c|c|c|c|c|}
\hline Studies & Acc. No. & MAC+T & Cell type & Infection & Platform \\
\hline 1 & GSE39889 & $4+4$ & Neutrophils & S. aureus & $\begin{array}{c}\text { GPL570 [HG-U133_Plus_2] } \\
\text { Affymetrix }\end{array}$ \\
\hline 2 & GSE13670 & $15+15$ & Macrophages & S. aureus & $\begin{array}{c}\text { GPL570 [HG-U133_Plus_2] } \\
\text { Affymetrix }\end{array}$ \\
\hline 3a & GSE13736 & $1+5$ & Endothelial cells & S. aureus & $\begin{array}{c}\text { GPL570 [HG-U133_Plus_2] } \\
\text { Affymetrix }\end{array}$ \\
\hline $3 \mathrm{~b}$ & GSE13736 & $1+5$ & Endothelial cells & S. aureus & $\begin{array}{c}\text { GPL570 [HG-U133_Plus_2] } \\
\text { Affymetrix }\end{array}$ \\
\hline $3 c$ & GSE13736 & $1+1$ & Endothelial cells & S. aureus & $\begin{array}{c}\text { GPL570 [HG-U133_Plus_2] } \\
\text { Affymetrix }\end{array}$ \\
\hline 4 & GSE16837 & $20+78$ & PMNL & S. aureus & $\begin{array}{c}\text { GPL570 [HG-U133_Plus_2] } \\
\text { Affymetrix }\end{array}$ \\
\hline 5 & GSE16129 & $10+42$ & PBMC & S. aureus & GPL96, 97 [HG-U133A] Affymetrix \\
\hline 6a & GSE44943 & $3+3$ & $\mathrm{HCCC}$ & S. aureus & [HuGene-1_0-st] Affymetrix Human \\
\hline $6 b$ & GSE44943 & $3+3$ & HCCC & S. aureus & [HuGene-1_0-st] Affymetrix Human \\
\hline Total & & $214 \mathrm{MA}$ & 21,632 Genes & & \\
\hline
\end{tabular}

GSE, Data series; MA, Microarray; C, Control; T, Treated; PMNL, Polymorphonuclear cells; PBMCs, Peripheral blood mononuclear cell, HCCC, Hepatocellular carcinoma cell line; S. aureus, Staphylococcus aureus; GPL, Gene platform

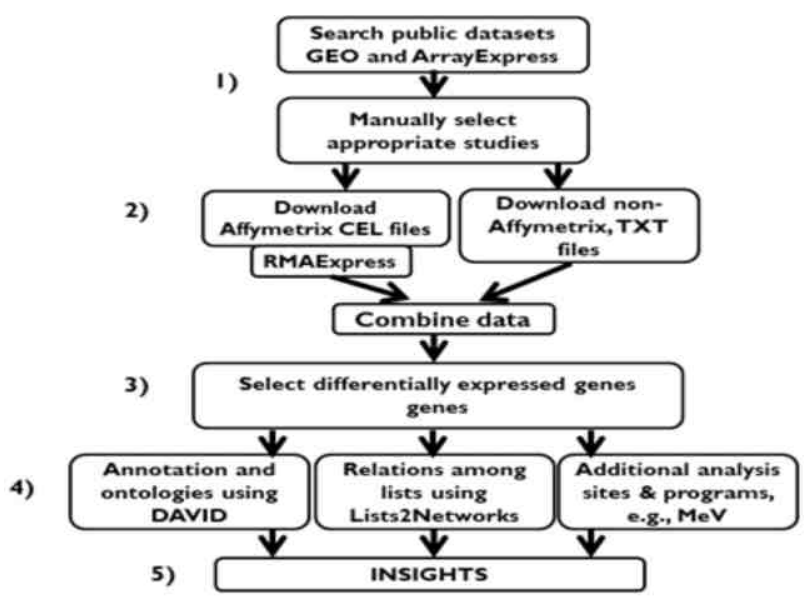

Fig 1: Flow chart of representative Meta-analysis procedure

Analysis of differential expression in cells challenged with inactivated $S$. aureus

Effects of UV- or heat-inactivated S. aureus were studied in bronchial epithelial and dendritic cells, 
respectively. This analysis included 2 studies, 3 data sets, 65 microarrays and 19,679 genes (Table 2).

\begin{tabular}{cccccc}
\hline \multicolumn{5}{c}{ Table 2: Data sets used in comparison of inactive $S$. aureus challenged vs. control cells } \\
\hline Studies & Acc. No. & MA C+T & Cell type & Infection & Platform \\
1 & GSE6802 & $3+2$ & Epithelial cells & Inactive S. aureus & GPL571 [HG-U133A_2] Affymetrix \\
2a & GSE44720 & $15+15$ & Dendritic cells & Inactive S. aureus & GPL10558 Illumina HumanHT-12 V4.0 \\
2b & GSE44720 & $15+15$ & Dendritic cells & Inactive S. aureus & GPL10558 Illumina HumanHT-12 V4.1 \\
Total & & 65 MA & 19,679 Genes & & \\
\hline
\end{tabular}

Differential expression analysis of $S$. aureus infected patients' blood

Three studies containing 3 data sets were found comparing differential gene expression in blood of $S$. aureus-infected patients and healthy controls. These studies used Affymetrix (GPL570, GPL571) and Illumina (GPL6947) platforms. After combining the data from three different studies, 279 microarrays and 15,001 common genes were obtained. In this analysis the study using Illumina (GPL6947) platform and 143 microarrays was modified before combining. The empty spaces in data were replaced with " 1 " to use in RankProd software, as described in Mimoso et al., $2014^{5}$ (Table 3).

\begin{tabular}{cccccc}
\hline \multicolumn{5}{c}{ Table 3: Data sets used in comparison of S. aureus infected patients vs. healthy controls } \\
\hline Studies & Acc. No. & MA C+T & Cell type & Infection & Platform \\
1 & GSE33341 & $43+32$ & Blood & S. aureus & GPL571 [HG-U133A_2] Affymetrix \\
2 & GSE25504 & $44+17$ & Blood & S. aureus & GPL570 [HG-U133_Plus_2] Affymetrix \\
3 & GSE30119 & $44+99$ & Blood & S. aureus & GPL6947 Illumina HumanHT-12 V3.0 \\
Total & & 279 MA & 15,001 Genes & & \\
\hline
\end{tabular}

Meta-analysis procedure

The data for transcriptional profiling were deposited in respective gene expression series as CEL or TXT files. These were processed for further analysis using RankProd software. RankProd software was used to identify the genes differentially expressed in $S$. aureus-challenged cells with $p$-values less than $10^{-2}$. RankProd analysis for each study group produced a table representing induced or suppressed genes in challenged cells. The DAVID software was used to get tables, charts and clusters for the induced or suppressed genes with $p$-value less than $10^{-4}$ obtained from RankProd output tables (Fig 1).

Comparison of live $S$. aureus infected vs. control cells

S. aureus can cause skin and systemic infections. ${ }^{6}$ We analyzed differential expression in endothelial cells, immune cells and hepatocellular carcinoma cell lines challenged with live $S$. aureus. The results for the

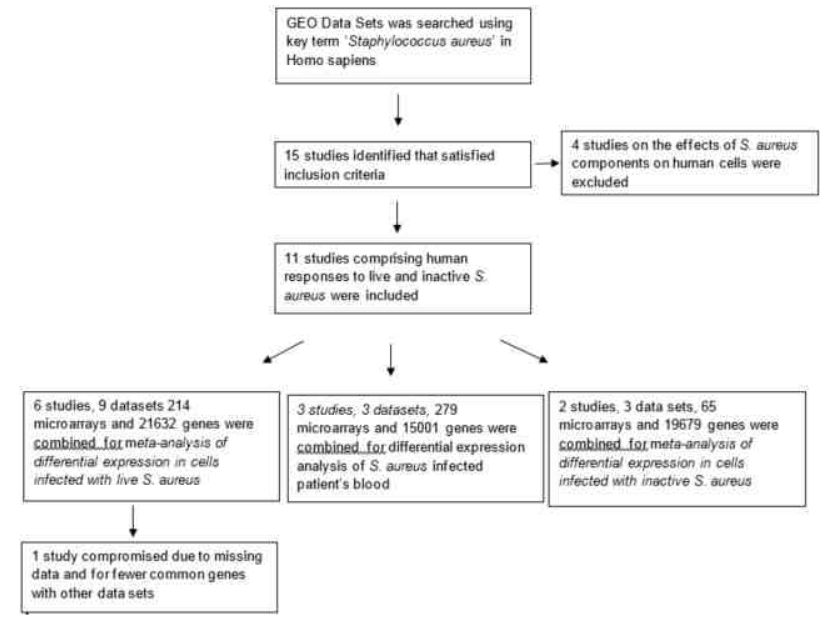

Fig 2: Prisma Statement for Meta- analysis

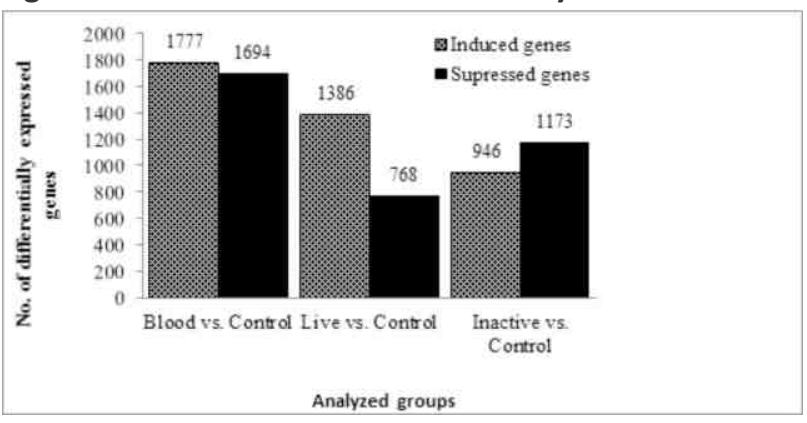

Fig 3: Number of differentially expressed genes in analyzed groups

comparison of ontological categories induced and suppressed in $S$. aureus infected cells are given in Table 4. We found that extracellular proteins, membrane receptors and intracellular genes that are involved in defense response were induced. Whereas intracellular processes including replication, gene expression, cell death and ubiquitin mediated protease pathway were suppressed.

The clusters containing overlapping ontological categories of genes induced in $S$. aureus-infected cells are presented in Table 4 . In the top regulated cluster the ontological categories including "Extracellular region part" and "extracellular space" were found (ES 14.45). Extracellular region part mainly included pattern recognition receptors (PRRs), cytokines, chemokines, hematopoietic, plasminogen and thrombospondins. In addition, genes involved in blood coagulation and thrombogenesis, such as inflammatory cytokines and chemokines, were found in this cluster. Interestingly, in cells challenged with live S. aureus, interleukin 1 receptor (IL-1R) and TLR specific MyD88-dependent pathway signaling molecules 
including mitogen-activated protein kinase (MAPK) and its regulators, which activate multiple processes in cells as gene expression, metabolism, cell division, cell morphology and cell survival, were largely induced. Anti-apoptosis genes were also upregulated.

In the $2^{\text {nd }}$ cluster ontological categories "plasma membrane part" and "intrinsic to plasma membrane" were found (ES 11.47). This cluster presented genes for receptors, specifically G-protein coupled receptors (GPCRs) that activate ligand specific secondary messengers for example, phosphatidylinositol-calcium, adenylyl cyclase 3 (ADCY3) and voltage-dependent potassium channels. In the $3^{\text {rd }}$ cluster ontological categories "response to wounding", "inflammatory response" and "defense responses" were found (ES 8.65). The genes present in this cluster were largely overlapping with the first cluster. We found that genes involved in activation of prothrombin complex following stable fibril formation, were uniquely expressed in this cluster. We also found genes for cell surface receptors including glycoprotein, voltage gated channels, tumor necrosis factor (TNF) receptor family, integrins and importantly TLR2. In the $4^{\text {th }}$ cluster (ES 6.42) ontological.

category "Positive regulation of biosynthesis" and "positive regulation of gene expression" are presented that included genes involved in the gene expression and replication.

In the $5^{\text {th }}$ cluster ontological categories "cell-cell signaling" and "synaptic transmission" were upregulated (ES 6.31). In the $6^{\text {th }}$ cluster (ES 5.72), we found gene ontologies "cell adhesion" and "cell-cell adhesion". In the $7^{\text {th }}$ cluster ontological categories "behavior" and "chemotaxis" were found (ES 5.59). We found the genes involved in cell to cell communication for example, calcium ion dependent transmembrane cell to cell junction, cadherin and proto-cadherins proteins in this cluster. Additionally, the genes for the proteins which bind to the cytoskeleton to carry cellular functions, including cell movement, cell division, endocytosis, and movement of organelles, were induced. The $8^{\text {th }}$ cluster upregulated in $S$. aureus-infected cells contained ontological categories "vasculature development" and "blood vessel development" (ES 5.45). We found that genes for transcription factors,

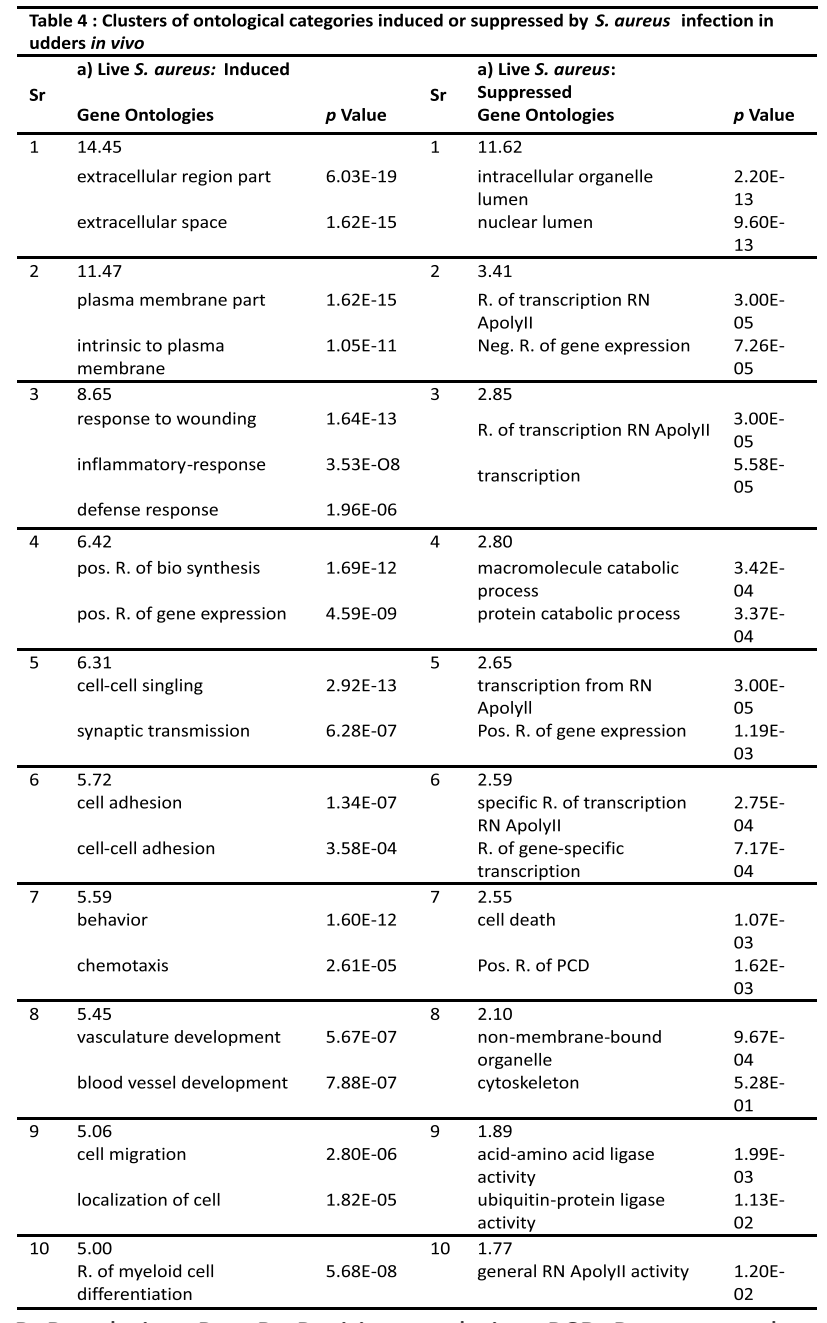

R. Regulation; Pos. R., Positive regulation; PCD, Programmed cell death

enzymes and signaling molecules were upregulated, they participate in transcription, replication and vasculature development processes. In the $9^{\text {th }}$ cluster the ontological categories "cell migration" and "localization of cell" were found (ES 5.06). Detailed study of these ontologies revealed genes contributing in the process of cytoskeleton interactions which play important function in mitosis and receptor-ligand interaction were upregulated. Similarly, downstream effector molecules that participate in the transmission of signals from tyrosine kinase receptors and small GTPases to the actin cytoskeleton were induced. In the $10^{\text {th }}$ cluster ontological category "regulation of myeloid cell differentiation" and "positive regulation of myeloid cell differentiation" were found (ES 5.00). The histone cluster and transcriptional factors were upregulated that specifically modulate the myeloid 
cell differentiation, possibly participating in formation of immune cells which provide defense response against external stimuli. The expression of proteins for normal cell processes, such as DNA repair, replication, cell division, development, might point the ability of $S$. aureus to decrease apoptosis of infected cells by upregulating cell processes. Moreover, processes e.g., apoptosis, cytoskeleton development to improve immunocytes taxis, receptors for bacterial components and reduced energy expenditure represented cells defense mechanisms against bacterial infection.

In table 4 the clusters of gene ontologies suppressed by live $S$. aureus infection in human cells are presented. In the top cluster (ES 11.62), gene ontologies such as "intracellular organelle lumen" and "nuclear lumen" were found. In S. aureusinfected cells defense, thrombus formation, immunity and chemotaxis and the gene expression processes were particularly suppressed. The genes found in clusters $1,2,3,5,6,9$ and 10 were overlapping and involved in the processes confined to nucleus, mitochondria and endoplasmic reticulum. These processes were related to the gene expression, protein processing and translocation. Nuclear receptor-mediated transcription was also repressed. We also found that the proteins for DNA modification, including histones, telomeres maintenance and DNA repair processes, were downregulated. The genes present in this cluster were completely different from the genes present in ontological category "positive regulation of gene expression" (cluster 4) in Table 5. The $2^{\text {nd }}\left(\right.$ ES 3.41) $3^{\text {rd }}$ (ES 2.85), $5^{\text {th }}$ (ES 2.65), $6^{\text {th }}$ (ES 2.59) and $10^{\text {th }}$ clusters (ES 1.77) again included genes that are involved in RNA polymerase II promoter dependent transcription process. In the $4^{\text {th }}$ cluster gene ontologies such as "macromolecule catabolic process" and "protein catabolic process" were found (ES 2.80). This cluster presented genes for protein catabolism via ubiquitin-proteasome pathway. Similarly, in the $9^{\text {th }}$ cluster we found proteins involved in ubiquitin-proteasome specifically involving ring finger proteins mediated proteolysis (ES 1.89). The $7^{\text {th }}$ cluster represented gene ontologies such as "cell death" and "positive regulation of programmed cell death" (ES 2.55). These ontologies included genes such as caspases that are necessary for the positive induction of cell death. Interestingly, we found the genes that modulate myeloid cells apoptosis. The proteins involved in mediating IFN $\gamma$ induced cell death were also suppressed in the $S$. aureus-infected cells. In the $8^{\text {th }}$ cluster cytoskeleton specific proteins were present in gene ontologies "non-membrane bound organelle" and "cytoskeleton" (ES 2.10). We found the proteins specific for microtubule cytoskeleton formation and regulation were suppressed. We have also found that proteins which may play an important role in integrin $\beta-1$ or $B$ cell receptor (BCR) mediated signaling in $\mathrm{B}$ - and T-cells were also suppressed.

\section{Comparison of heat inactivated $S$. aureus challenged vs. control cells}

The live $S$. aureus and inactivated $S$. aureus challenged cells present different sets of differentially expressed genes. ${ }^{7}$ Therefore, we have compared differential expression of heat inactivated

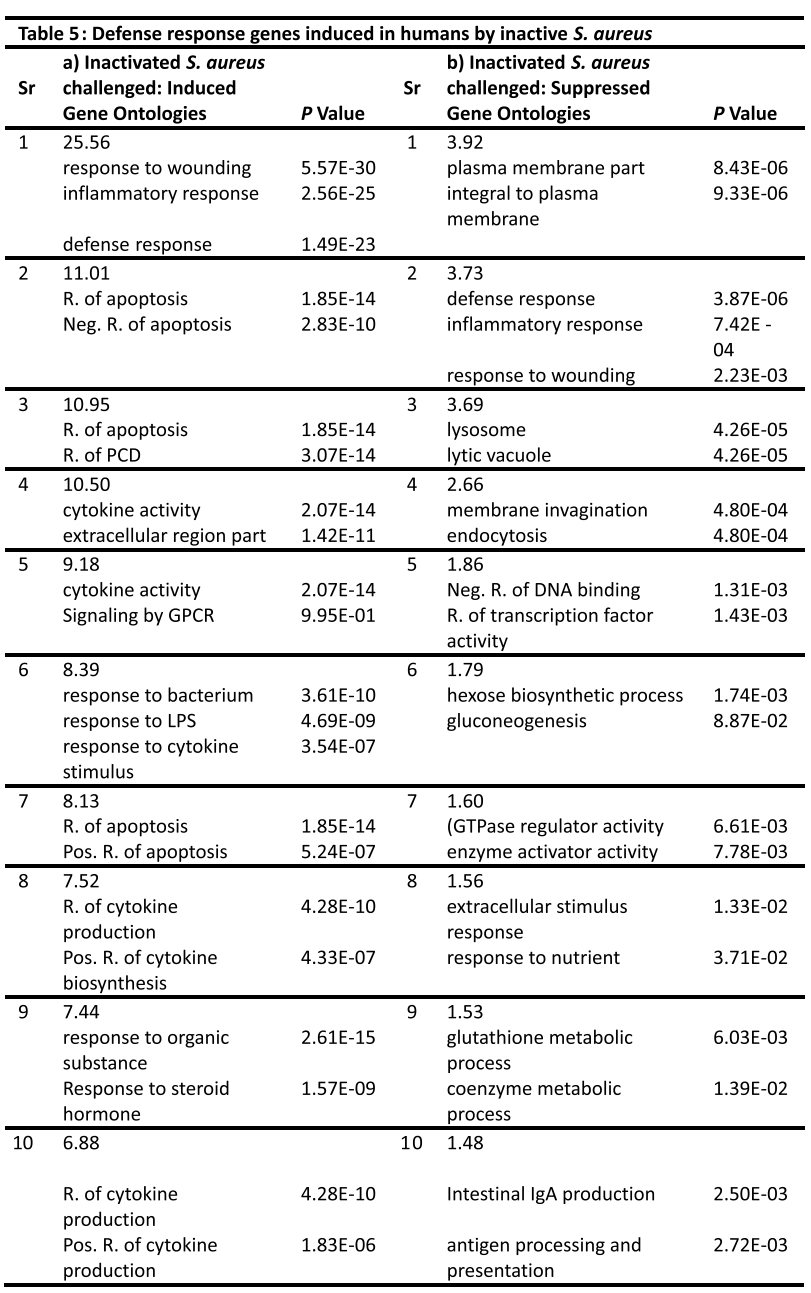

R. Regulation; Pos. R., Positive regulation; Neg. R., Negative regulation; LPS, Lipopolysaccharide; PCD, Programmed cell death 
S. aureus challenged versus control cells using Metaanalysis.

The clusters of gene ontologies induced in heatinactivated $S$. aureus challenged cells are given in Table 5. We found that three major processes were induced including defense response, regulation of apoptosis and cytokine production.

We found that the $1^{\text {st }}$ (ES 25.56), $6^{\text {th }}$ (ES 8.39) and $9^{\text {th }}$ (ES 7.44) clusters presented genes for defense mechanisms against bacterium. The ontological categories such as "response to wounding", "inflammatory response" and "defense response" are present in the top regulated cluster (ES 25.56) as observed in Table 3 and 4. Like previous comparisons in $S$. aureus-challenged vs. control cells, we found that innate immunity specific Gram positive bacteria receptor present on cell surface, TLR2, or general bacterial receptor TLR8 found in intracellular endosomes $^{8}$ were upregulated. Interestingly, proteins for MyD88-dependent TLR response pathway were induced. In contrast to live $S$. aureus challenged cells where innate immunity specific chemoattractant i.e. myeloid cells activators were overrepresented, in inactive $S$. aureus chemoattractant for innate as well as adaptive immunity specific cells were induced. Specially, a number TNF- $\alpha$ complex mediated macrophagesspecific cytokines and co-stimulatory cluster of differentiation (CD) molecules, that share immune response by macrophages internalization, cell adhesion and T cell receptor (TCR) binding, were induced. In wounding response platelet derived growth factors and gene clusters were upregulated. Proteins involved in receptor-mediated endocytosis and regulators of apoptosis were also found in this cluster. The $6^{\text {th }}$ cluster presented the signaling mechanisms in "response to bacterium" and "response to molecules of bacterial origin" (ES 8.39). The innate immune receptor TLR2 and immune response genes including chemokines ( $\mathrm{C}-\mathrm{C}$ motif) cytokines including IL-1B, IL-6, IL-10, and Interleukin1 receptor-associated kinase 3 (IRAK3), cytokines regulators were upregulated. Additional transcription and nuclear factors (FOS, JUN and NFKB1A) that participate in extrinsic cell death processes were also upregulated. In $9^{\text {th }}$ cluster, genes for the response systems to endogenous peptide hormone stimulus were induced (ES 7.44). We observed that most of the calcium stimulated pathways involved in metabolism and signaling to peptide hormone leading to initiation of cell death pathways were induced.

In $2^{\text {nd }}$ (ES 11.01), $3^{\text {rd }}$ (ES 10.95) and $7^{\text {th }}$ (ES 8.13) clusters; negative regulators of apoptotic pathways, that lead to increased cell survival were observed. Specifically, downstream signaling molecules for NLRs were induced. The major genes complexes which regulate anti-cell death processes were induced in this cluster including TNF- $\alpha$ and associated proteins, ILs (1, 6 and 10), DNA repair proteins, nuclear factors, oncogenes, transcription activators and serine threonine kinases. Cell cycle regulation proteins stimulated by MAPK pathways were also found in this cluster. Cytoskeletal proteins involved in cell communication, attachment and locomotion were also induced.

In the $4^{\text {th }}$ (ES 10.50), $5^{\text {th }}$ cluster (ES 9.18), $8^{\text {th }}$ (ES 7.52) and $10^{\text {th }}$ (ES 6.88) clusters genes involved in cytokine production and regulation were found. In chemokines and cytokines involved in defense against extracellular stimulus dominating the adaptive immune cells were found. In addition, growth regulators and proteins involved in local inflammatory process were induced. In addition, signaling pathways which use $G$ protein-coupled receptors (GPCR) after activation by prostaglandins, adrenomedullin and immune cells were induced.

The clusters representing the gene ontologies for suppressed genes in $S$. aureus challenged human cells are given in Table 5. The top cluster included ontological categories such as "plasma membrane part" and "integral to plasma membrane". In this cluster the genes for adaptors and receptors largely associated with adaptive immune system, including lymphocytes and natural killer cells were found. A large number of the antigen-presenting proteins (CD molecules) were also suppressed. Additionally, adaptive immunity specific antigen processing proteins for $\mathrm{MHCll}$ family were downregulated. Adhesion molecules, dendritic cells specific genes were downregulated. Th1 regulators were also suppressed. ATP dependent constitutive or basal glucose transporters and some nucleoside transporters were downregulated. Calciummediated signaling molecules, $\mathrm{G}$ protein receptors, adaptors such as ADCY, and chemokines 
participating in cell proliferation, were found in this cluster. The receptors for Gram negative bacteria and immunoglobulin E (IgE) Fc fragments were also suppressed.

In the $2^{\text {nd }}$ cluster (ES 3.73) the ontological categories "defense", and "inflammation" related processes were presented. In detail, study showed that signaling pathways linked to Gram negative bacteria specific cell receptors (TLR4, TLR5) were presented. Lymphocyte specific antigen proteins, some myeloid and neutrophil specific proteins that bind TLR4 receptor to mediate downstream signaling were downregulated. In addition, MYD88 that acts as an adaptor downstream of TLR2 and TLR4 receptors was repressed. In the $3^{\text {rd }}$ cluster proteins associated with "lysosome" involved in degradation of protein were represented (ES 3.69). These included CD proteins, $\mathrm{MHCll}$ complex which specify antigen presenting cells recognition and major enzymes important in degradation of the targeted molecule such as cathepsins, catalases, lipases and peptidases. The the $4^{\text {th }}$ cluster presented ontological categories such as "membrane invagination" and "endocytosis" (ES 2.66). Interestingly, we found that the genes present in this cluster were related to endocytosis and intracellular processing of clathrin coated vesicle, which suggested that genes involved in phagocytosis of foreign bodies were downregulated. In $5^{\text {th }}$ (ES 1.86) and $6^{\text {th }}$ (ES 1.79) clusters of S. aureus challenge downregulated genes, the genes for "negative regulation of DNA binding" i.e. transcription process were principally found. The repressed genes were mostly DNA damage-induced transcriptional regulator and cytokine production genes. Surprisingly, we found that carbohydrate metabolism genes were downregulated.

In the $7^{\text {th }}$ cluster (ES 1.60), we found that GTPase-Ras mediated signaling pathway proteins, that participate in endocytosis after binding with cations e.g., zinc, were downregulated. In the $8^{\text {th }}$ cluster ontological category "response to extracellular stimuli" and "nutrient" was found (ES 1.56). These ontological categories included organic substance response genes that lead to activation of stress response, wound healing proteins. In addition, proteins for cell death regulation were also downregulated. In the $9^{\text {th }}$ cluster the "glutathione metabolic process" and "coenzyme metabolic process" ontologies were represented (ES 1.53). Study of the genes in these clusters showed that enzymes processing glutathione were downregulated. In the $10^{\text {th }}$ cluster antibody production and antigen presentation and processing activity is represented (ES 1.48). We found that in this cluster $\mathrm{MHCll}$ antigen presenting and processing proteins as well as adhesion proteins were presented.

Comparison of transcriptional profiles of blood from $S$. aureus infected patients vs. healthy controls S. aureus is an important human pathogen which causes local as well as systemic infections. The study of infected blood transcriptome analysis should provide valuable information about systemic changes in molecular expression of leukocytes during infection that can be used for rapid diagnosis and therapeutic purposes. ${ }^{9}$ In present study, the transcriptional microarray data from blood of infected neonates and adults was combined and analyzed to identify the differentially expressed genes in S. aureus infected versus healthy controls through Meta-analysis (Table 6).

The clusters of gene ontologies induced in S. aureus infected human blood are given in Table 6 . Principally, we found that genes for bacterial components recognition receptors and downstream processing pathways including internalization, processing and inflammatory responses were induced.

In the comparison of differential expression of genes in blood of $S$. aureus infected patients and controls, we found that the ontological categories "defense response", "response to wounding" and "inflammation" are present in the top regulated cluster as observed in previous comparisons (ES 20.21). Innate immunity specific PRR's for Gram positive bacteria including TLRs and NLRs were upregulated in blood of infected patients. Acute inflammatory response genes including complement components, co-stimulatory molecules as CD55 and CD163, macrophages specific phagocytic receptor and associated enzymes were induced. The chemoattractant released from innate immunocytes and specific for adaptive immunocytes were upregulated. IL-18 released from antigen presenting cells and cytokines which affect thymus as well as proliferation of T-cells were also found. In addition, 
IL-25 was induced which may lead to MyD88dependent activation of NF-KB and also required for cytokine production and response mediation. Innate immunity specific phagocytosis, immune cells activators and thrombospondin, important in increasing movement of vesicle inside the cell, were also upregulated.

The $2^{\text {nd }}$ (ES 10.57). $3^{\text {rd }}$ (ES 7.80), $4^{\text {th }}(E S 6.86), 6^{\text {th }}$ (ES $5.29)$ and $8^{\text {th }}$ (ES 5.11) clusters included genes for PRRs, TLRs (1, 2, 5, 6 and 8), sodium-chloride dependent neurotransmitter symporters.

\begin{tabular}{|c|c|c|c|c|c|}
\hline $\mathrm{Sr}$ & $\begin{array}{l}\text { a) S. aurenus infected } \\
\text { blood: Induced } \\
\text { Gene Ontologies }\end{array}$ & $P$ Value & $\mathrm{Sr}$ & $\begin{array}{l}\text { b) S. aureus infected } \\
\text { blood: Suppressed } \\
\text { Gene Ontologies }\end{array}$ & $P$ Value \\
\hline \multirow[t]{4}{*}{1} & 20.21 & & 1 & 9.98 & \\
\hline & defense response & $3.00 \mathrm{E}-25$ & & $\begin{array}{l}\text { translational } \\
\text { elongation }\end{array}$ & $9.54 \mathrm{E}-19$ \\
\hline & response to wounding & 3.39E-20 & & Gene Expression & 3.09E-09 \\
\hline & inflammatory response & $2.23 \mathrm{E}-17$ & & & \\
\hline 2 & $\begin{array}{l}10.57 \\
\text { intrinsic to plasma membrane } \\
\text { integral to plasma membrane }\end{array}$ & $\begin{array}{l}3.41 \mathrm{E}-13 \\
4.78 \mathrm{E}-13\end{array}$ & 2 & $\begin{array}{l}7.92 \\
\text { lymphocyte activation } \\
\text { hemopoiesis }\end{array}$ & $\begin{array}{l}3.66 \mathrm{E}-17 \\
4.87 \mathrm{E}-12\end{array}$ \\
\hline 3 & $\begin{array}{l}7.80 \\
\text { cell fraction } \\
\text { membrane fraction }\end{array}$ & $\begin{array}{l}4.19 \mathrm{E}-09 \\
2.34 \mathrm{E}-08\end{array}$ & 3 & $\begin{array}{l}6.28 \\
\text { organelle lumen } \\
\text { nuclear lumen }\end{array}$ & $\begin{array}{l}8.23 \mathrm{E}-09 \\
3.80 \mathrm{E}-06\end{array}$ \\
\hline \multirow[t]{2}{*}{4} & $\begin{array}{l}6.86 \\
\text { response to bacterium }\end{array}$ & 4.26E-10 & 4 & $\begin{array}{l}5.66 \\
\text { R. of lymphocyte } \\
\text { activation }\end{array}$ & $2.99 \mathrm{E}-13$ \\
\hline & response to LPS & $8.48 \mathrm{E}-06$ & & $\begin{array}{l}\text { R. of leukocyte } \\
\text { activation }\end{array}$ & $3.40 \mathrm{E}-12$ \\
\hline \multirow[t]{2}{*}{5} & $\begin{array}{l}5.79 \\
\text { hemostasis }\end{array}$ & $1.94 \mathrm{E}-07$ & 5 & $\begin{array}{l}5.21 \\
\text { Graft-versus-host } \\
\text { disease }\end{array}$ & 3.09E-11 \\
\hline & $\begin{array}{l}\text { Complement \& coagulation } \\
\text { cascades }\end{array}$ & $1.78 \mathrm{E}-06$ & & $\begin{array}{l}\text { Cell adhesion } \\
\text { molecules }\end{array}$ & $2.32 \mathrm{E}-07$ \\
\hline \multirow[t]{3}{*}{6} & 5.29 & & & $\begin{array}{l}\text { Antigen processing } \\
\text { and presentation }\end{array}$ & $6.16 \mathrm{E}-07$ \\
\hline & innate immune response & $4.50 \mathrm{E}-13$ & 6 & 4.81 & \\
\hline & adaptive immune response & $3.35 \mathrm{E}-09$ & & $\begin{array}{l}\text { T cell receptor } \\
\text { complex }\end{array}$ & $1.46 \mathrm{E}-07$ \\
\hline \multirow[t]{2}{*}{7} & $\begin{array}{l}5.12 \\
\text { vesicle }\end{array}$ & $1.94 \mathrm{E}-08$ & 7 & $\begin{array}{l}\text { R. of Ca signaling } \\
4.22\end{array}$ & $2.93 \mathrm{E}-04$ \\
\hline & Secretory granule & $1.77 \mathrm{E}-07$ & & $\begin{array}{l}\text { plasma membrane } \\
\text { part }\end{array}$ & $1.37 \mathrm{E}-06$ \\
\hline \multirow[t]{4}{*}{8} & 5.11 & & & $\begin{array}{l}\text { intrinsic to plasma } \\
\text { membrane }\end{array}$ & $332 \mathrm{E}-05$ \\
\hline & $\begin{array}{l}\text { response to organic } \\
\text { substance }\end{array}$ & $1.80 \mathrm{E}-07$ & 8 & 3.89 & \\
\hline & $\begin{array}{l}\text { response to hormone } \\
\text { stimulus }\end{array}$ & $1.51 \mathrm{E}-05$ & & $\begin{array}{l}\text { RNA complex } \\
\text { biogenesis }\end{array}$ & $2.36 \mathrm{E}-05$ \\
\hline & & & & $\begin{array}{l}\text { rRNA metabolic } \\
\text { process }\end{array}$ & $1.15 \mathrm{E}-04$ \\
\hline \multirow[t]{2}{*}{9} & $\begin{array}{l}5.09 \\
\text { membrane organization }\end{array}$ & $2.09 \mathrm{E}-06$ & 9 & $\begin{array}{l}3.76 \\
\text { Pos. R. of apoptosis }\end{array}$ & $2.73 E-06$ \\
\hline & phagocytosis & $2.85 \mathrm{E}-05$ & & Pos. R. of PCD & $3.40 \mathrm{E}-06$ \\
\hline \multirow[t]{3}{*}{10} & 4.49 & & 10 & 3.50 & \\
\hline & protein dimerization activity & $5.90 \mathrm{E}-07$ & & $\begin{array}{l}\text { lymphocyte } \\
\text { proliferation }\end{array}$ & $2.18 \mathrm{E}-05$ \\
\hline & homodimerization activity & $7.39 \mathrm{E}-05$ & & $\begin{array}{l}\text { mononuclear cell } \\
\text { proliferation }\end{array}$ & $3.65 \mathrm{E}-05$ \\
\hline
\end{tabular}

R. Regulation; Pos. R., Positive regulation; Neg. R., Negative regulation; LPS, Lipopolysaccharide; PCD, Programmed cell death belonging to solute carrier (SLC)16 and SLC6 superfamily and MyD88-dependent signaling pathways. We found that genes that are involved in identification and downstream processing of extracellular particles were principally induced. Scavenger receptors for acetylated low density lipoprotein (LDL), which also have role in angiogenesis, were upregulated. Inflammatory cytokines and their receptors (IL-18R, IL-1 $\alpha$ ) and genes released from activated antigen presenting innate immune cells that might be involved in activation of B-cells were induced. The negative regulators of cell death and calcium binding process were also induced in S. aureus-infected patients. These inflammatory cytokines are also key activators of humoral and cellular immunity.

Interestingly, in the $5^{\text {th }}$ cluster gene ontologies such as "hemostasis" and "complement and coagulation cascade" were upregulated (ES 5.79). The genes included in this cluster were mostly involved in hemostasis process, concentrating immune cells density at site of injury. The upregulated gene complexes included coagulation factors, platelet glycoproteins, plasminogen activators and thrombomodulins. In addition, calcium binding proteins which accelerate ATP mediated fibrin formation process were induced. In $7^{\text {th }}$ (ES 5.12) and $9^{\text {th }}$ (ES 5.29) clusters genes for transport of lipoprotein particle by membrane invagination and vesicle formation were induced. Cytoskeleton and calcium transport genes involved in the process of translocation were also induced.

The $10^{\text {th }}$ cluster presented gene ontologies such as "protein dimerization activity" and "homodimerization activity" (ES 4.49). We found that proteins involved in process of gene expression were largely upregulated; principally RNA polymerase II-dependent transcription was induced. In addition, transcription regulators as well as apoptosis mediators were induced. Similarly, cofactors $\left(\mathrm{Ca}^{+2}\right.$ and $\left.\mathrm{Zn}^{+2}\right)$ mediated guanylate cyclase and GTP derived energy process involved in processing of pathogen containing cells were induced.

The top cluster (ES 9.98) of the genes downregulated in S. aureus-infected patients included ontological categories "translational elongation" and "gene expression" (Table 6). These categories revealed that cytosolic and nucleic ribosomal proteins were the major suppressed genes in the top cluster. In addition, some translation elongation factors were also suppressed. Surprisingly, in contrast to 
transcription of immunity specific genes induced in the infected patients, the ribosomal proteins transcription was largely suppressed. Similarly, the $3^{\text {rd }}$ cluster (ES 6.28) presented the organelle lumen and nuclear lumen. The proteins for cell cycle progression, adaptive immune cells activation genes, glucose metabolism and positive regulation of cell death genes were also downregulated. The the $8^{\text {th }}$ cluster (ES 3.89) again presented genes involved in nuclear processes such as formation of ribosomal RNA.

In the $2^{\text {nd }}\left(\right.$ ES 7.92), $4^{\text {th }}$ (ES 5.66) and 10th (ES 3.50) clusters adaptive immunity specific genes involved in lymphocytes activation, proliferation, differentiation and regulation, involving both $\mathrm{B}$ - and T-lymphocytes were found. Co-stimulatory and adhesion molecules and accessory proteins present downstream in T-cell receptor signaling pathways which subsequently activate the two processes were found in suppressed genes: 1) expression of certain cytokines that enhance proliferation, differentiation and immune response 2) and downregulation of ubiquitin mediated proteolysis. In summary, The critical genes for the activation of helper T cell, Th1 (IFN $\gamma$ ) and Th2 (IL-4) lymphocytes, such as CD40LG and IL4, were also repressed.

In the $5^{\text {th }}$ cluster ontological categories such as "graft versus host disease", "cell adhesion molecules" and "antigen processing and presentation" were presented (ES 5.21). The genes involved in maturation of $\mathrm{MHCll}$ molecules that leads to survival of natural killer (NK) cells in response to infectionmediated activation were found in this cluster. $\ln 6^{\text {th }}$ cluster gene ontologies including "T-cell receptor complex" and "Regulation of calcium signaling" were found (ES 4.81). Proteins which have probable role in assembly and expression of the TCR complex as well as signal transduction upon antigen triggering were also downregulated.

The the $7^{\text {th }}$ cluster presented gene ontologies such as "plasma membrane part" and "intrinsic to plasma membrane part" (ES 4.22). The genes present in these categories were plasma membrane integral proteins involved in adaptive immunity activation. Furthermore, a large number of the genes and processes related to adaptive immunity including IL2 regulators, lymphocytes attractants, IL receptors $(1,2,5,7)$ stimulated Jak-stat signaling molecules, metal ion transporters $\left(\mathrm{Ca}^{+2}, \mathrm{Na}^{+1}, \mathrm{~K}^{+1}\right)$, active T-cells survival proteins, $\mathrm{T}$-cell mediated mitogen-activated protein kinase (MAPK) pathway gens, and genes for IFN $\gamma$ synthesis were suppressed. In $9^{\text {th }}$ cluster (ES 3.76) ontological categories "positive regulation of apoptosis" and "Positive regulation of programmed cell death" were presented. The positive regulator of apoptosis, apoptosis-related signaling pathways and NKs activated formation of toxicity proteins were found here.

\section{Discussion}

The live $S$. aureus induced genes for both innate and adaptive immunity, where platelet function and vasodilation dominated the immune response. Moreover, MyD88-dependent signaling pathways were induced. Interestingly, unlike previous analyses live $S$. aureus did not modulate the apoptotic process as a cytoprotective strategy; but upregulated genes for normal cellular processes for example gene expression, neuron signaling and importantly mitotic cell cycle. This survival strategy was already reported in obligatory bacteria, which survive either by stimulating cell processes thus providing infected human cells resistance to the apoptosis stimulus, or directly regulate apoptosis machinery. ${ }^{10}$ The downregulated processes included gene expression, ubiquitin mediated protein catabolism and IFN $\gamma$ mediated cell death processes. Furthermore, transcription process of innate immune response genes was induced whereas this process was suppressed for other cell processes. ${ }^{11,12}$

The differential expression in the cells challenged with inactivated S. aureus was slightly different from live $S$. aureus challenged ones as revealed from the Meta-analysis. Similar to live $S$. aureus, positive induction of cellular processes such as cell cycle and growth regulation was also seen in inactivated $S$. aureus challenged cells proposing the comparable cytoprotective mechanism. Importantly, in inactivated $S$. aureus challenged cells carbohydrate biosynthesis process was downregulated whereas this process was upregulated in live $S$. aureus infected cells. Further MyD88 signaling pathway was induced by live $S$. aureus infection while suppressed by inactivated $S$. aureus treatment thus defining different cellular defense strategies against live and inactive S. aureus (Table 5).,13

Interestingly, the results for Meta-analysis of 
differential expression in the blood of infected patients were marginally different from live- and inactivated- $S$. aureus challenged cells. MyD88independent signaling pathway was induced unlike the active $S$. aureus mediated responses where MyD88-dependent signaling pathway was more prominent. Moreover, we observed that membrane receptors including symporters, aquaporin's and solute carriers were over-represented in this analysis. Similar to the live- and inactivated-S. aureus analysis, innate immunity and negative regulators of cell death were induced while adaptive immunity and positive regulators of cell death were downregulated (Table 6). ${ }^{9,14,15}$

In the live $S$. aureus challenged cells, innate immunity, adaptive immunity and wounding process genes were induced whereas transcription of genes for normal cell processes, protein catabolism and apoptosis were suppressed by the $S$. aureus infection. Similarly, Koziel and co-workers found a significant increase in the expression of antiapoptotic genes by transcriptome analysis of human monocyte-derived macrophages during $S$. aureus infection. ${ }^{6}$ We also observed that in the $S$. aureus-infected patients the genes for defense against bacterial infection, innate immunity, solute receptors and anti-apoptotic processes were upregulated, whereas gene expression, adaptive immunity and positive regulators of apoptosis were downregulated. In contrast, Smith et al, reported that in infected neonates, innate immune-negative feedback opposes innate immune activation while suppression of T-cell co-stimulation is coincident with selective upregulation of CD85 co-inhibitory pathways. ${ }^{15}$

Like activated $S$. aureus, in heat-inactivated $S$. aureus challenged cells innate and adaptive immune responses to molecules of bacterial origin, cell cycle, growth regulators, negative regulators of cell death as well as genes for blood thinning were induced. Further, among the suppressed one's genes for adaptive immunity, antibody production, antigen presentation and processing were found. However, for apoptosis process nuclear receptors for intrinsic cell death and calcium mediated signaling and apoptosis were induced. Glucose metabolism process was induced in live $S$. aureus challenged cells it was suppressed by inactivated S. aureus. Further
MyD88 signaling pathway was induced by live $S$. aureus infection whereas suppressed by inactivated S. aureus treatment. Mayer et al., found that regulation of the TLR expression by host cells serves as a mechanism for adjusting sensitivity of microbial recognition in different compartments thus limiting infection. In contrast, Smith et al., found that $S$. aureus infection develop an elevated set-point of myeloid regulatory signaling and sugar-lipid metabolism with concomitant inhibition of lymphoid responses in host cells. ${ }^{15}$

\section{Conclusion}

Meta-analysis of microarray data from $S$. aureuschallenged cells indicated that innate immune process genes were induced largely while adaptive immunity genes were suppressed. In addition, this Meta-analysis revealed cytoprotective strategies adopted by $S$. aureus to evade host immune system mediated bactericidal activity. In this context, live $S$. aureus and inactivated $S$. aureus induced cellular processes and suppressed apoptosis genes. Similarly, apoptosis was suppressed in the infected patient's blood by upregulating anti-apoptotic genes. This review increased our understanding of the human molecular responses to infection and cytoprotective strategies adopted by the $\mathrm{S}$. aureus to evade host immune systems.

\section{Acknowledgments}

We acknowledge financial support from Higher Education Commission, Islamabad, Pakistan, and from the R.O. Perelman Department of Dermatology, NYU Medical School, New York, USA.

\section{REFERENCES}

1. Moran GJ, Krishnadasan A, Gorwitz RJ, Fosheim GE, McDougal LK, Carey RB, et al. Methicillin-resistant S. aureus infections among patients in the emergency department. $\mathrm{N}$ Engl J Med. 2006; 355: 666-74.

2. Duhen T, Geiger R, Jarrossay D, Lanzavecchia A, Sallusto F. Production of interleukin 22 but not interleukin 17 by a subset of human skin-homing memory $T$ cells. Nat Immunol. 2009; 10: 857-63.

3. Cho JS, Pietras EM, Garcia NC, Ramos RI, Farzam DM, Monroe HR, et al. IL-17 is essential for host defense against cutaneous Staphylococcus aureus infection in mice. J Clin Invest. 2010; 120: 1762-73.

4. Miller LS, Cho JS. Immunity against Staphylococcus aureus cutaneous infections. Nat Rev Immunol. 2011; 11: 505-18.

5. Mimoso C, Lee DD, Zavadil J, Tomic-Canic M, Blumenberg $M$. Analysis and meta-analysis of transcriptional profiling in human epidermis. Methods Mol Biol. 2014; 1195: 61-97. 
6. Koziel J, Maciag-Gudowska A, Mikolajczyk T, Bzowska M, Sturdevant DE, Whitney AR, et al. Phagocytosis of Staphylococcus aureus by macrophages exerts cytoprotective effects manifested by theupregulation of antiapoptotic factors. PLoS One. 2009; 4: e5210.

7. Banchereau R, Baldwin N, Cepika AM, Athale S, Xue Y, Chun IY, et al. Transcriptional specialization of human dendritic cell subsets in response to microbial vaccines. Nature communications. 2014; 5:1-4.

8. Bergstrøm B, Aune MH, Awuh JA, Kojen JF, Blix KJ, Ryan L, et al. TLR8 Senses Staphylococcus aureus RNA in Human Primary Monocytes and Macrophages and Induces IFN- $\beta$ Production via a TAK1-IKKß-IRF5 Signaling Pathway. J Immunol. 2015; 195: 1100-11.

9. Banchereau R, Jordan-Villegas A, Ardura M, Mejias A, Baldwin $\mathrm{N}, \mathrm{Xu} \mathrm{H}$, et al. Host immune transcriptional profiles reflect the variability in clinical disease manifestations in patients with Staphylococcus aureus infections. PLoS One. 2012; 7: e34390.

10. Häcker G, Kirschnek S, Fischer SF. Apoptosis in infectious disease: how bacteria interfere with the apoptotic apparatus. Med Microbiol Immunol. 2006; 195: 11-9.

11. Stark L, Matussek A, Strindhall J, Geffers R, Buer J, Kihlström $E$, et al. Staphylococcus aureus isolates from blood and anterior nares induce similar innate immune responses inendothelial cells. APMIS. 2009; 117: 814-24.

12. Matussek A, Strindhall J, Stark L, Rohde M, Geffers R, Buer J, et al. Infection of human endothelial cells with Staphylococcus aureus induces transcription of genes encoding aninnate immunity response. Scand J Immunol. 2005; 61: 536-44.

13. Mayer AK, Muehmer M, Mages J, Gueinzius K, Hess C, Heeg $K$, et al. Differential recognition of TLR-dependent microbial ligands in human bronchial epithelial cells. The Journal of Immunology. 2007; 178: 3134-42.

14. Ahn SH, Tsalik EL, Cyr DD, Zhang Y, van Velkinburgh JC, Langley RJ, et al. PLoS One. 2013; 8: e48979.

15. Smith $C L$, Dickinson $P$, Forster $T$, Craigon $M$, Ross $A$, Khondoker MR, et al. Identification of a human neonatal immune-metabolic network associated with bacterial infection. Nature communications. 2014; 5: 1-5. 